\title{
Hybrid compressor design for a 10PW laser
}

\author{
D. Kramer ${ }^{1}$, J. Novák ${ }^{1,2}$, and B. Rus ${ }^{1}$ \\ ${ }^{1}$ Institute of Physics, Czech Academy of Science, Na Slovance 2, 18221 Praha 8, Czech Republic \\ ${ }^{2}$ Faculty of Nuclear Science and Physical Engineering, CTU in Prague, Břehová 7, 11519 Prague 1, Czech Republic
}

\begin{abstract}
The ELI beamlines facility will house a 10PW laser with ultrashort pulse duration. The laser damage threshold of the coatings limits the minimum size of the last compression grating. Due to production restrictions, the last grating has to be tiled. This design was produced in order to minimize the total number of gratings in the compressor together with the spatial requirements.
\end{abstract}

\section{Introduction}

One of the aims of the ELI-beamlines [1] facility is to build a 10PW laser chain and thus allow reaching unprecedented intensities in the experiments.

There are several possible approaches to produce a $10 \mathrm{PW}$ laser beam. If one produces a compressed beam with a few hundreds of joules, its pulse length must not exceed several tens of fs. Another possible approach consists in compressing a beam with energy above a $\mathrm{kJ}$ and compressing it to nearly $100 \mathrm{fs}$. This approach is more favourable for the acceleration of electrons beyond the energy of few $\mathrm{GeV}$. In such case, the pulse length for acceleration should be in the order of few hundred fs as indicated in [2].

While possible solution for compressing the $\mathrm{kJ}$ pulses is the conventional symmetrical configuration proposed by Treacy [3], its implementation in this case calls for tiling of the gratings due currently available maximum size of $\sim 960 \mathrm{~mm}$. The design presented in this paper makes an attempt to find a feasible alternative solution of a kJ class compressor, with a minimized number of required gratings as well as minimum spatial requirements.

\section{Design specifications}

The conventional design of the compressor would consist of 2 symmetric pairs of gratings with equal compressive powers. The size of the gratings would be driven by the damage threshold of the last grating, where the pulse is the shortest.

The symmetry of the compressor is preferred as the second pair of gratings eliminates the spatial chirp produced by the first one. However, according to [4], a non-symmetric solution of a compressor could be found as well.

By setting the compressive power of the first grating pair lower by choosing a smaller groove density, one can reach smaller incidence angles. In that way the size of the gratings will decrease and the fluence on their surface will increase. Main aim of this design is to reduce a number of gratings used in first half of the compressor, where pulses are still relatively long, and therefore also laser induced damage threshold (LIDT) is higher.

\subsection{Beam parameters}

The design assumes a beam produced by an amplification chain made of Silicate and Phosphate Nd glasses. Such approach produces a sufficient spectral bandwidth to reach a transform limit of 126fs. The corresponding central wavelength was set to $1053.5 \mathrm{~nm}$. The stretched pulse duration is set to $2.5 \mathrm{~ns}$ (FWHM). The input pulse energy used in the calculations is $1900 \mathrm{~J}$. This energy is reduced by $5 \%$ after each reflection on a grating. The beam parameters are summarized in Table 1.

Table 1. Beam parameters used in the design of the compressor.

\begin{tabular}{|l|l|}
\hline Central wavelength & $1053.5 \mathrm{~nm}$ \\
\hline Spectral width FWHM / full & $13 \mathrm{~nm} / 26 \mathrm{~nm}$ \\
\hline Stretched pulse duration & $2.5 \mathrm{~ns}$ \\
\hline Compressed pulse duration & $126 \mathrm{fs}$ \\
\hline Max grating length $(1 \& 2)$ & $960 \mathrm{~mm}$ \\
\hline Input energy & $1900 \mathrm{~J}$ \\
\hline Beam size & $400 \times 400 \mathrm{~mm}^{2}$ \\
\hline Beam profile & Super-Gaussian, G=20 \\
\hline Grating diffraction efficiency & $95 \%$ \\
\hline $\begin{array}{l}\text { Max fluence at 130fs on the } \\
\text { last grating surface }\end{array}$ & $390 \mathrm{~mJ} / \mathrm{cm}^{2}$ \\
\hline
\end{tabular}

This is an Open Access article distributed under the terms of the Creative Commons Attribution License 2.0, which permits unrestricted use, distribution, and reproduction in any medium, provided the original work is properly cited. Article available at http://www.epj-conterences.org or http://dx.doi.org/10.1051/epjcont/20134800010 


\section{Compressor design}

The input parameters mentioned above result in the target value of the group delay dispersion (GDD) of $-1.136 \times 10^{-8}$ $\mathrm{fs}^{2}$ in order to compress the pulse. This value is calculated assuming a Gaussian shape of the stretched and compressed pulse according to formula (1)

$$
G D D=\frac{T_{0}}{4 \ln 2} \sqrt{T_{1}^{2}-T_{0}^{2}}
$$

where $T_{0}$ and $T_{1}$ are the pulse durations of the compressed and the stretched pulse.

The current production limit in ultrafast grating size is close to $1 \mathrm{~m}$. However for this design, the maximum length of a single grating was set to a more conservative $960 \mathrm{~mm}$. The last 2 gratings have to be tiled in order to stay below the required fluence.

\subsection{Damage threshold}

In a symmetrical system, the two pairs of gratings would be identical, therefore also tiled. The asymmetrical approach allows using a different grating constant for each pair and eventually avoiding tiling of the first pair.

Available measurements [5] of LIDT of large MultiLayer Dielectric (MLD) gratings show that for the 3.2ps long pulses the damage occurred at $3.9 \mathrm{~J} / \mathrm{cm}^{2}$ (and 4.7 $\mathrm{J} / \mathrm{cm}^{2}$ for a second sample) beam normal. In order to deduce the LIDT for the fs pulse durations, the power scaling with exponent 0.33 according to [6] was used. The resulting fluence is $1.39 \mathrm{~J} / \mathrm{cm}^{2}$ assuming the pulse duration of $130 \mathrm{fs}$. This design took a $10 \%$ lower fluence as a target value $\left(1.25 \mathrm{~J} / \mathrm{cm}^{2}\right)$. The fluence on the grating surface $\left(72^{\circ}\right)$ therefore should not be higher than 390 $\mathrm{mJ} / \mathrm{cm}^{2}$. Further measurements are required in order to confirm this value, which may be too optimistic.

\subsection{Design targets}

Given the rather complicated set of requirements, it was not possible to find a direct solution with zero residual spatial chirp. The design was executed as an optimization task with the spatial chirp as one of the variables to be minimized. The above mentioned requirements together with the goal to keep the compressor as compact as possible, are giving the following design targets:

-the $2^{\text {nd }}$ grating smaller than $960 \mathrm{~mm}$

-the vertical separation between gratings $>20 \mathrm{~mm}$

-the $3^{\text {rd }}$ grating smaller than $2 * 960 \mathrm{~mm}$

-the angle of incidence on $3^{\text {rd }}$ grating $>72^{\circ}$

-the GDD of the system at $-1.136 \times 10^{-8} \mathrm{fs}^{2}$

The final optimization was performed in the ZEMAX optical design code. The GDD of the compressor is a part of the design targets. Therefore it had to be calculated directly in ZEMAX. This was executed thanks to a macro described in [7].

The grating constants were set as free parameters as the current technologies permit to vary considerably the grating constant for the Multilayer Dielectric Gratings.

\subsection{Resulting design}

The design was inspired by [4], where only a small difference between the grating constants was used in order to minimize the footprint of the compressor. However in this design, the asymmetry has to be much larger to permit smaller angles of incidence on the first grating pair.

The main parameters of the optimized design are summarized in Table 1. The first and second gratings can be made of one piece; the other two must be tiled from two pieces. This would require apodization of the middle part of the beam to cover the gap between the last grating tiles.

The resulting spatial chirp of $17 \mathrm{~mm}$ is reasonably small for a $400 \mathrm{~mm}$ beam size, while the compressor is very compact as can be seen in figure 2 .

Table 1: Resulting parameters of the compressor.

\begin{tabular}{|l|l|}
\hline Grating const 1,2 & $1328 \mathrm{lpmm}$ \\
\hline Grating const 3,4 & $1687 \mathrm{lpmm}$ \\
\hline Angle of incidence $\theta_{1}$ & $47.811^{\circ}$ \\
\hline Angle of incidence $\theta_{3}$ & $72.009^{\circ}$ \\
\hline GR1 to GR2 & $4712 \mathrm{~mm}$ \\
\hline GR2 to GR3 & $3950 \mathrm{~mm}$ \\
\hline GR3 to GR4 & $4459 \mathrm{~mm}$ \\
\hline Vertical separation GR1 GR2 & $20 \mathrm{~mm}$ \\
\hline Residual spatial chirp (max) & $17 \mathrm{~mm}$ \\
\hline GR1 length & $596 \mathrm{~mm}$ \\
\hline GR2 length & $884 \mathrm{~mm}$ \\
\hline GR3 length & $1920.8 \mathrm{~mm}$ \\
\hline GR4 length & $1313 \mathrm{~mm}$ \\
\hline Third order dispersion & $9.1910^{8} \mathrm{fs}^{3}$ \\
\hline Astigmatism & $<\lambda / 30$ \\
\hline
\end{tabular}

Another important factor for asymmetric gratings is the resulting astigmatism of the compressed beam. This effect was simulated within ZEMAX with the Physical Optics Propagation module and was found to be very low with a maximum of $\lambda / 30$ as shown in figure 1 . The beam was modeled with a circular footprint.

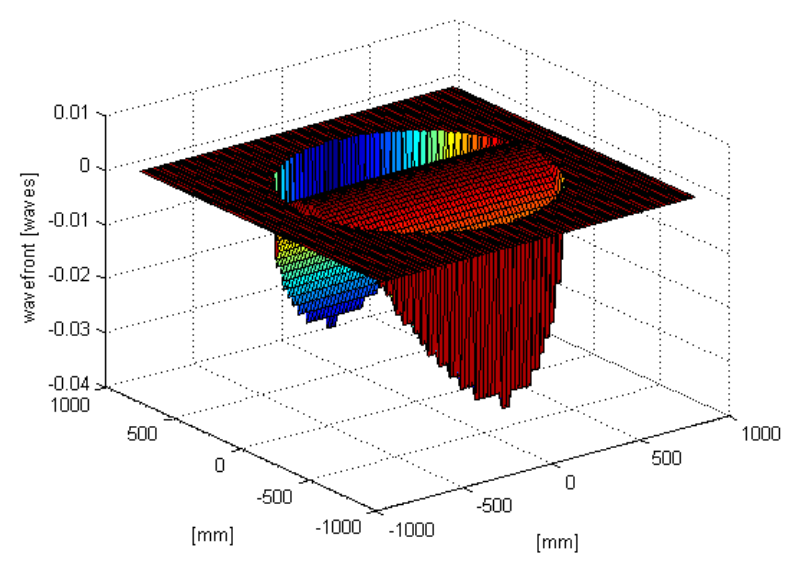

Fig. 1. Wavefront error simulated with Physical Optics Propagation tool in the ZEMAX code.

Table 3 lists the incident fluences on all the gratings and the corresponding pulse durations. The fluences normal to the beam are listed for reference only. 


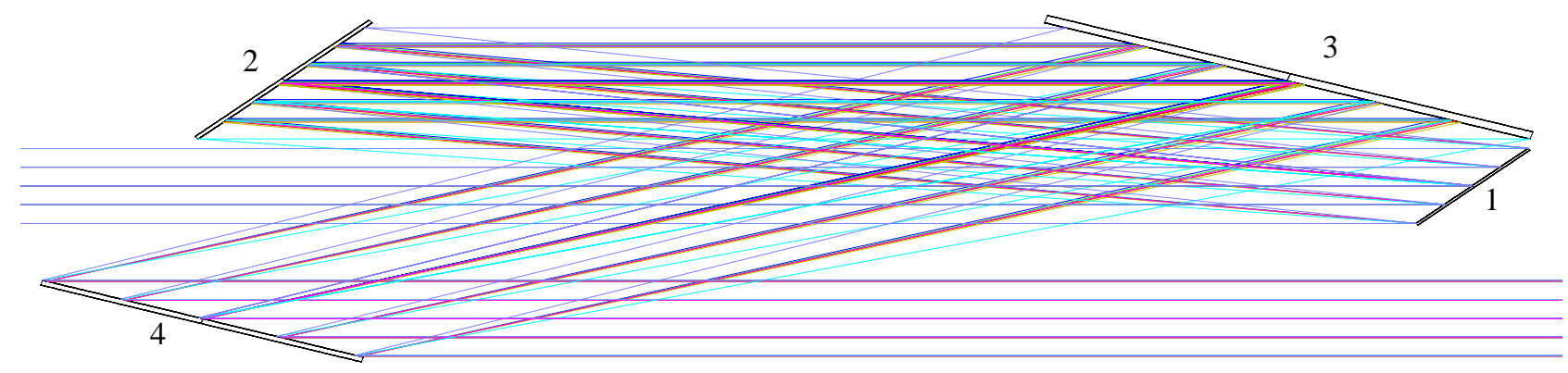

Fig. 2. Mixed-grating compressor layout with spatial chirp compensation. Thicknesses of the gratings are not to scale. Different colours represent the spectral width of $26 \mathrm{~nm}$.

The pulse length is reduced on the first grating pair by the GDD of $-3.03 \times 10^{-7} \mathrm{fs}^{2}$ down to $1840 \mathrm{ps}$. For this pulse length the fluence of $0.93 \mathrm{~J} / \mathrm{cm}^{2}$ is still well below the damage threshold. On the second grating pair the pulse is shortened by the GDD of $-8.33 \times 10^{-7} \mathrm{fs}^{2}$ to $130 \mathrm{fs}$. In this case, the fluence of $0.39 \mathrm{~J} / \mathrm{cm}^{2}$ is reached on the fourth grating.

\section{Discussion}

The grating constant for the first pair was determined to be 1328 lines per $\mathrm{mm}$. This proves to be well beyond the possibilities of the current MLD grating production capabilities. Such grating design can be reached with the Gold technology. This however turns out to be impossible, as the MLD gratings have to work with $s$ polarization and Gold gratings with $p$-polarization.

The solution for this problem could be the hybrid technology gratings. They are combining multiple dielectric layers with the metallic layers. The efficiency of such designs can be virtually independent on the polarization state as shown in [8]. Nevertheless, these technologies require further development in order to reach commercial grade. Most importantly, the damage threshold of such gratings has to be thoroughly investigated. Even if the gratings will be subject only to ns pulses, the fluences are still quite high and the angle of incidence rather modest.

Table 2: List of fluences incident on the individual gratings of the compressor for a 10PW laser (input energy $1900 \mathrm{~J}$ ).

\begin{tabular}{|l|l|c|c|}
\hline Surface & \multicolumn{1}{|c|}{$\begin{array}{c}\text { Incident } \\
\text { fluence } \\
{\left[\mathbf{J} / \mathbf{c m}^{2}\right]}\end{array}$} & $\begin{array}{c}\text { Pulse } \\
\text { duration } \\
{[\mathbf{p s}]}\end{array}$ \\
\hline GR1 & Peak at $0^{\circ}$ incid. & 1.46 & 2500 \\
\hline GR1 & Peak at $47.8^{\circ}$ incid. & 0.98 & 2500 \\
\hline GR2 & Peak at $0^{\circ}$ incid. & 1.39 & 1840 \\
\hline GR2 & Peak at $47.8^{\circ}$ incid. & 0.93 & 1840 \\
\hline GR3 & Peak at $0^{\circ}$ incid. & 1.32 & 1840 \\
\hline GR3 & Peak at $72^{\circ}$ incid. & 0.41 & 1840 \\
\hline GR4 & Peak at $0^{\circ}$ incid. & 1.25 & 0.130 \\
\hline GR4 & Peak at $72^{\circ}$ incid. & 0.39 & 0.130 \\
\hline Mirr. & Peak at $0^{\circ}$ incid. & 1.19 & 0.130 \\
\hline Mirr. & Peak at $45^{\circ}$ incid. & 0.84 & 0.130 \\
\hline
\end{tabular}

The fluence of $1.25 \mathrm{~J} / \mathrm{cm}^{2}$ seems to be rather optimistic and further measurements are necessary to verify the damage threshold of the MLD gratings. Such value would likely be too close to the damage threshold and leave a very small margin for the amplitude modulations of the laser beam. One option would be the extension of the beam size along the grooves close to $600 \mathrm{~mm}$. This would not impact the length of the gratings nor the compressor.

The zero order reflection from the $3^{\text {rd }}$ grating will reflect onto the $1^{\text {st }}$ one. This does not seem to impact the functionality of the compressor. Though, alignment procedures and diagnostics layout have to be defined.

\section{Conclusions}

Using an asymmetric compressor design allows decreasing the number of gratings for a kJ level $10 \mathrm{PW}$ class laser, which will be constructed in the ELI Beamlines facility. It could reduce the cost of the compressor and the complexity of the first grating pair. At the same time the design is very compact and therefore a smaller vacuum compressor vessel could be used.

Nevertheless, the first grating pair requires a hybrid metal-multilayer dielectric technology, which still needs time to mature into a verified commercial product with proven damage threshold and homogenous quality throughout the active area. At the same time, the Laser Induced Damage Threshold of the MLD gratings would have to be improved in order to permit this design. Otherwise the beam would have to be asymmetrically enlarged along the grooves causing the subsequent beam transport to be challenging.

\section{Acknowledgment}

We would like to thank Douglas Smith from Plymouth Grating Laboratory for very helpful discussions. We acknowledge the support of the following EU grants CZ.1.05/1.1.00/02.0061 and CZ.1.07/2.3.00/20.0091.

\section{References}

1. B. Rus et al., Proc. of SPIE, 8080, 10 (2011)

2. S. F. Martins, R. A. Fonseca, W. Lu, W. B. Mori, L. O. Silva, Nature Physics, 6 (2010)

3. E. B. Treacy, IEEE J. of Quant. Elect. 5, 454 (1969)

4. D.N. Fittinghof, B. Wattelier, C.P.J. Barty, UCRLJC-152484, (2003)

5. Douglas J. Smith, Mike McCullough, Claire Smith, Takuya Mikami, Takahisa Jitsuno, Proc. of SPIE, 7132 (2008) 
6. M. Mero, J. Liu, W. Rudolph, D. Ristau, K. Starke, Phys. Rev. B 71, 11 (2005)

7. D. C. O'Shea, Appl. Opt. 45, 19 (2006)

8. A. Hu, C. Zhou, H. Cao, J. Wu, J. Yu, W. Jia, Appl. Opt. 51, 20 (2012) 